\title{
Simultaneous dual-gas QEPAS detection based on a fundamental and overtone combined vibration of quartz tuning fork
}

\author{
Hongpeng Wu, ${ }^{1,2}$ Xukun Yin, ${ }^{1,2}$ Lei Dong, ${ }^{1,2, a)}$ Kailong Pei, ${ }^{1,2}$ Angelo Sampaolo, ${ }^{3,4}$ \\ Pietro Patimisco, ${ }^{3,4}$ Huadan Zheng, ${ }^{1,2}$ Weiguang Ma, ${ }^{1,2}$ Lei Zhang, ${ }^{1,2}$ Wangbao Yin, ${ }^{1,2}$ \\ Liantuan Xiao, ${ }^{1,2}$ Vincenzo Spagnolo, ${ }^{3}$ Suotang Jia, ${ }^{1,2}$ and Frank K. Tittel ${ }^{4}$ \\ ${ }^{1}$ State Key Laboratory of Quantum Optics and Quantum Optics Devices, Institute of Laser Spectroscopy, \\ Shanxi University, Taiyuan 030006, China \\ ${ }^{2}$ Collaborative Innovation Center of Extreme Optics, Shanxi University, Taiyuan 030006, China \\ ${ }^{3}$ Dipartimento Interateneo di Fisica, Università degli Studi di Bari and Politecnico di Bari, CNR-IFN BARI, \\ Via Amendola 173, Bari 70126, Italy \\ ${ }^{4}$ Department of Electrical and Computer Engineering, Rice University, Houston, Texas 77005, USA
}

(Received 23 January 2017; accepted 8 March 2017; published online 21 March 2017)

\begin{abstract}
A dual-gas quartz-enhanced photoacoustic spectroscopy (QEPAS) sensor system based on a frequency division multiplexing technique of a quartz tuning fork (QTF) was developed and experimentally demonstrated. Two beams from two independently modulated lasers are focused at two different positions between the QTF prongs to excite both the QTF fundamental and 1st overtone flexural modes simultaneously. The $2 f$-wavelength modulation technique is employed by applying two sinusoidal dithers, whose frequencies are equal to a half of the QTF fundamental and 1st overtone frequencies, respectively, to the currents of two excitation lasers. The resonance frequency difference between two flexural modes ensures that the correlated photoacoustic signals generated by different target gases do not interfere with each other. The proposed QEPAS methodology realizes a continuous real-time dual-gas monitoring with a simple setup and small sensor size compared with previous multi-gas QEPAS sensors. Published by AIP Publishing. [http://dx.doi.org/10.1063/1.4979085]
\end{abstract}

Multi-gas on-line detection is a topic of considerable interest in recent years because of its important applications in environmental monitoring, medical diagnosis, industrial process control, food industry, and pollution monitoring. ${ }^{1-4}$ Photoacoustic spectroscopy (PAS), which offers many advantages in terms of sensitivity, selectivity, and stability is a widely recognized technique for multi-gas analysis. ${ }^{5-7}$ In the traditional PAS technique, modulated excitation light is selectively absorbed by a target gas inside the photoacoustic cell and results in the generation of an acoustic wave by nonradiative energy relaxation processes. Trace gas concentration levels can be obtained by detecting the intensity of the acoustic wave via a broadband microphone. ${ }^{7}$ Quartz enhanced photoacoustic spectroscopy (QEPAS) is a modification of the traditional PAS, in which a quartz tuning fork (QTF) is utilized as a sharp acousto-electrical transducer, instead of broadband microphones, to detect the photoacoustic excitation, thus providing a robust, compact, and costeffective sensor. ${ }^{8-15}$

A QEPAS sensor requires a judicious design to perform chemical analysis of a multi-component gas mixture due to the fact that a force sensing based QTF cannot recognize the molecular source of an induced acoustic wave. To date, three different configurations of multi-gas QEPAS sensors have been reported. The first design employs an acoustic detection module (ADM) and a single laser source with an emission spectral range capable to cover all the selected absorption lines of the target gases. ${ }^{16}$ As a result, this type of QEPAS sensor cannot be used to detect multi-gas species when there are large spectral gaps between their target absorption lines,

${ }^{\text {a)} E l e c t r o n i c ~ m a i l: ~ d o n g l e i @ s x u . e d u . c n ~}$ due to the wavelength tuning limitation of a single laser source. To address this issue, a second approach has been proposed, in which several laser sources resonant with absorption lines belonging to different target gases are used in conjunction with a single QEPAS ADM. Optical switches are equipped to control these laser beams to pass in turn through the QEPAS ADM. ${ }^{17}$ The two abovementioned configurations are based on a time division multiplexing (TDM) technique and hence cannot offer continuous realtime multi-gas monitoring. A third straightforward approach for real-time, multi-gas detection is to integrate several QEPAS sensors into one. ${ }^{18}$ Each sensor targets a different gas. However, this makes the sensor complex, bulky, and not cost-effective.

Since 2013, custom made QTFs with few $\mathrm{kHz}$ fundamental resonance frequencies $f_{0}$ have been reported for QEPAS trace-gas detection. ${ }^{19,20}$ These custom QTFs have a similar geometrical design to a standard QTF but are larger in size and with the prongs spaced by up to $1 \mathrm{~mm}$, thereby facilitating optical alignment. Furthermore, the lower $f_{0}$ values of the custom QTFs are not only beneficial to detect gases with slow vibration-translation (V-T) relaxation rates, such as NO, $\mathrm{CO}$, and $\mathrm{CH}_{4},{ }^{17,21}$ but also allow the implementation of their 1 st overtone flexural modes for QEPAS trace-gas detection. ${ }^{22}$ It has been demonstrated theoretically and experimentally that the custom QTF behaves like a standard QTF transducer in terms of mechanical properties and electricity properties. $^{20-22}$ The standard QTF can be replaced with a custom QTF in a QEPAS sensing system, maintaining the compact size and the robustness of the system. To-date, all QEPAS sensors reported in the literature operate in either the fundamental or 1 st overtone flexural modes. ${ }^{20-22}$ In this letter, we 
report a dual-gas QEPAS sensing approach based on a QTF frequency division multiplexing (FDM) technique. The QTF in a dual-gas QEPAS sensor is excited simultaneously in the fundamental and 1st overtone flexural modes by two independently modulated lasers. The two target gases are detected via demodulation of the custom QTF piezoelectric signal at the fundamental frequency $f_{0}$ and the 1 st overtone frequency $f_{1}$, respectively, by means of two lock-in amplifiers (LIAs). This approach overcomes the limitations existing in previous multi-gas QEPAS sensors and results in a compact and costeffective sensor architecture.

A QTF in the form of a two-pronged fork with the prongs connected at one end can be modeled as two single cantilevers when the coupling between the two prongs can be neglected. ${ }^{23}$ The resonance frequencies $f_{\mathrm{n}}$ of the QTF nth flexural mode can be expressed as

$$
f_{n}=K \times \frac{T}{L^{2}} \times n^{2},
$$

where $n=1.194$ for the fundamental flexural mode and $n=2.988$ for the 1 st overtone flexural mode, $K$ is a constant which is determined by the QTF material (Young modulus and density), $T$ is the prong width, and $L$ its length, as schematically depicted in Fig. 1(a). ${ }^{21,22}$ It has been demonstrated theoretically and experimentally that the nth-overtone flexural mode can be considered as $n$-coupled point-masses subsystems, each one located at an antinode. ${ }^{21}$ As a result, the exciting laser beam must be focused at an antinode in order to maximize the QEPAS signal. The simulated motions of the fundamental and 1st overtone flexural modes are shown in Figs. 1(b) and 1(c), respectively. A combined vibration of both flexural modes mentioned above was also simulated, as shown in Fig. 1(d), which can be implemented by placing two independently modulated beams at the corresponding antinodes to excite simultaneously both flexural modes, thus providing two detection channels of the fundamental and 1st overtone frequencies. The custom QTF employed in this work is $\sim 4.6$ times larger than the standard $32 \mathrm{kHz}-\mathrm{QTF}$ and has a prong length-to-width ratio of 17 . The distance between the single antinode of the fundamental mode and the second antinode of the overtone mode is $9 \mathrm{~mm}$, large enough to accommodate two laser beams for independent mode excitation for dual-gas sensing measurements. ${ }^{22}$

A schematic of the sensor setup is shown in Fig. 2. Two DFB lasers (DL1 and DL2) were used as excitation sources for the fundamental and 1st overtone flexural modes, respectively. The lasers were mounted onto two driver boards (DB1 and DB2), which can control the temperature and current of both lasers. Ramp signals generated from the DB1 and DB2 driver boards are used to scan the two laser wavelengths across the corresponding target absorption lines. Furthermore, two sine wave signals generated from two function generators (FG1 and FG2) modulate the wavelengths of the DL1 and DL2 at half of the fundamental frequency $f=f_{0} / 2$ and 1 st overtone frequency $f^{\prime}=f_{1} / 2$, respectively, for second harmonic (2f) detection. ${ }^{24,25}$

The QTF was enclosed inside a gas cell with two $\mathrm{CaF}_{2}$ windows for optical access. The DL1 and DL2 laser beams were focused down to $\sim 0.25 \mathrm{~mm}$ on the QTF plane, using
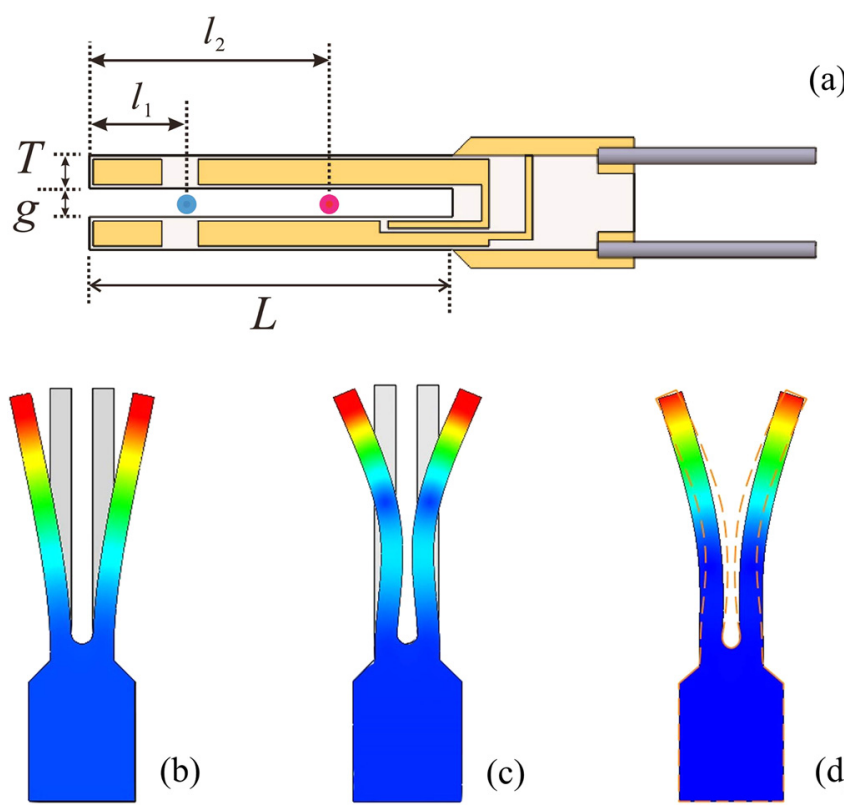

(b)
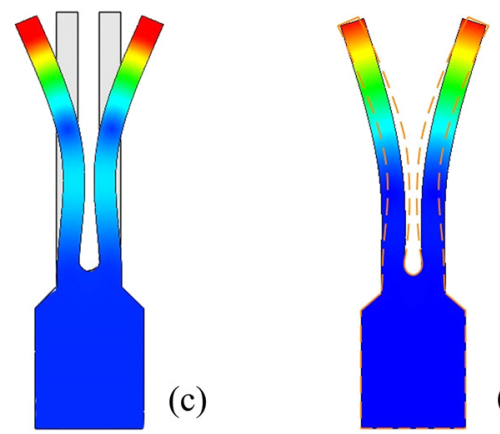

(d)

FIG. 1. (a) Custom QTF with two exciting laser beams. $T=1 \mathrm{~mm}$ is the prong width. $L=17 \mathrm{~mm}$ is the prong length and $\mathrm{g}=0.7 \mathrm{~mm}$ is the spacing between the prongs. The red and blue circles are the positions of the two exciting laser beam spots. $l_{l}\left(l_{2}\right)$ is the distance from the custom QTF opening to the two laser beam circles (blue and red). (b)-(d) Illustrations representing the deformation of the QTF prongs vibrating in the fundamental flexural mode, the 1st overtone flexural mode, and the combined vibration of both modes, respectively. The illustrations were obtained using SolidWorks software. The original shape of the QTF prongs appears in gray in (b) and (c). The gold dashed line in (d) represents the QTF prong deformation when the QTF is excited only in the 1st overtone flexural mode. A color scale was used ranging from blue to red, representing the deformation degree of the QTF prongs.

two fiber collimators (OZ optics, Ltd., Model LPC-01). The DL1 beam was positioned at $l_{1}=2 \mathrm{~mm}$ below the QTF opening near to the antinode point for the fundamental flexural mode, while the DL2 beam was positioned at $l_{2}=9.5 \mathrm{~mm}$ below the QTF opening close to the most efficient 1st overtone flexural mode antinode point. ${ }^{22}$ The QTF and the fiber



FIG. 2. Schematic of a dual-gas QEPAS sensor system based on a QTF combined vibration in fundamental and 1 st overtone modes. TA: transimpedance amplifier. 
collimators were fixed on the same holder to ensure the position stability of the laser beam with respect to the QTF. The QTF was connected to a custom transimpedance amplifier (TA), which delivered the amplified signal to two LIAs (LIA1 and LIA2) (Stanford Research Systems, Model SR830) for $2 f$ detection. The electrical parameters of the two LIAs were the same, a $12 \mathrm{~dB} /$ oct filter slope and a $300 \mathrm{~ms}$ time constant, except for the demodulation frequencies which were $f_{0}$ and $f_{1}$, respectively.

The DL1 and DL2 can be both tuned to a wavelength of $1368.6 \mathrm{~nm}$, resonant with a water vapor absorption line. The two lasers were used to detect the same $\mathrm{H}_{2} \mathrm{O}$ absorption line in order to verify that there is no interference effect between the fundamental and 1st overtone flexural modes, when the QTF was operated in the combined vibration. The experiment was performed using a gas mixture composed of $1.6 \%$ water vapor in $\mathrm{N}_{2}$, at a pressure of 700 Torr. With this condition, the $f_{0}$ and $f_{1}$ of the QTF were $2,868 \mathrm{~Hz}$ and $17741 \mathrm{~Hz}$, respectively. The selected $\mathrm{H}_{2} \mathrm{O}$ absorption line is located at $7306.75 \mathrm{~cm}^{-1}$ with a line intensity of $1.8 \times 10^{-20} \mathrm{~cm} \cdot \mathrm{mol}^{-1} \cdot{ }^{26}$ The two lasers were tuned to the selected absorption line by setting the laser temperature. The output power of the DL1 and the DL2 was 21.2 $\mathrm{mW}$ and $19.6 \mathrm{~mW}$, respectively.

The $2 f$ spectral scans obtained from the dual-gas QEPAS sensor for three different QTF vibration conditions are depicted in Fig. 3. Figure 3(a) shows the signals measured by LIA1 at $f_{0}$ when the QTF operates in the pure fundamental flexural mode ( $\boldsymbol{\Delta}$ symbols) and when both fundamental and 1st overtone modes are simultaneously excited ( symbols). The overlap of the two curves indicates that when the QTF oscillates in the fundamental mode, the simultaneous excitation of the 1st overtone mode does not affect the fundamental QEPAS signal, i.e., the fundamental flexural mode is independent of the 1 st overtone flexural mode. A similar

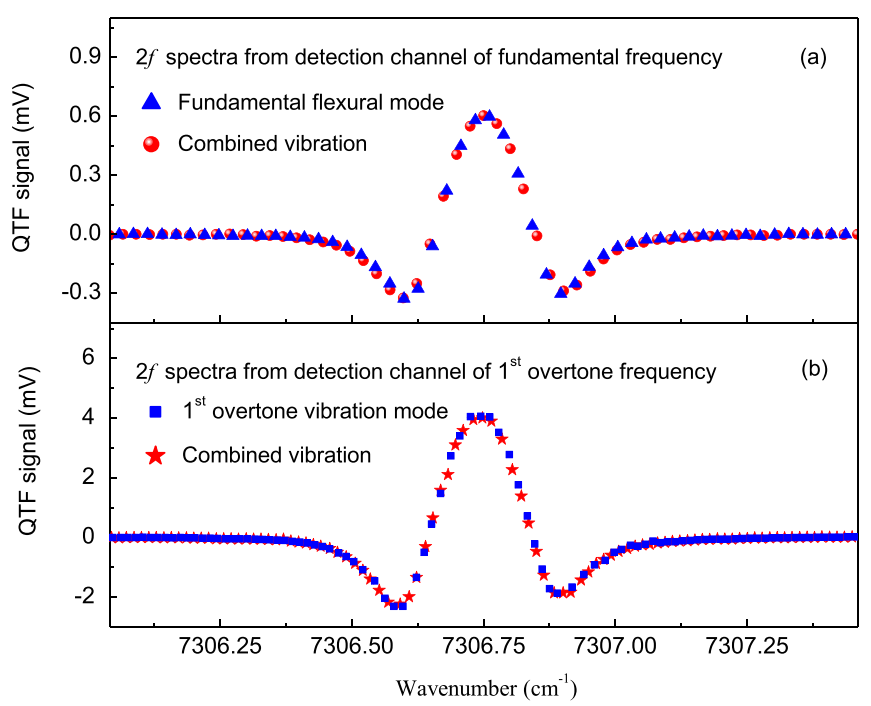

FIG. 3. QEPAS spectral scans obtained for a $1.6 \% \mathrm{H}_{2} \mathrm{O}: \mathrm{N}_{2}$ gas mixture at a pressure of 700 Torr, with the QTF vibrating in different modes. (a) Triangles and circles represent $2 f$ spectra measured by the detection channel of the fundamental frequency, when the QTF operated in the pure fundamental flexural mode and fundamental-1st overtone combined vibrational motion, respectively. (b) Rectangles and stars represent $2 f$ spectra measured by the detection channel of the $1^{\text {st }}$ overtone frequency, when the QTF operated in the pure 1st overtone vibration mode and fundamental-1st overtone combined vibrational motion, respectively. conclusion was obtained by analyzing the signals measured by LIA2 at $f_{1}$, shown in Fig. 3(b). When the QTF operates in the 1st overtone flexural mode, no interference effects are observed if the QTF fundamental mode is simultaneously excited. Therefore, both the fundamental and 1st overtone QTF signals can be separately extracted when both modes are simultaneously excited.

To assess the performance of the dual-gas QEPAS sensor, a noise-level analysis was performed. The QEPAS sensor noise from the detection channel of the fundamental frequency was measured when both QTF modes are simultaneously excited. The lasers wavelengths were locked onto the target absorption line and the gas cell was filled with pure $\mathrm{N}_{2}$ at 700 Torr. A $1 \sigma$-noise level of $1.85 \mu \mathrm{V}$ was observed, comparable with $1.80 \mu \mathrm{V}$ obtained when the QTF was excited only in the fundamental mode, as shown in Fig. 4. A similar result was also obtained for the detection channel of the 1st overtone frequency. Therefore, no excess noise was observed when the QTF operates in the combined vibration motion with respect to the pure fundamental or 1 st overtone flexural mode for both detection channels.

To demonstrate the capability of the QEPAS sensor for simultaneous dual-gas spectral detection, the DL2 was replaced with a DFB laser (DL3) for acetylene $\left(\mathrm{C}_{2} \mathrm{H}_{2}\right)$ detection in the sensor. A $\mathrm{C}_{2} \mathrm{H}_{2}$ absorption line located at $6541.96 \mathrm{~cm}^{1}$ with a line-strength of $3.5 \times 10^{-20} \mathrm{~cm} \cdot \mathrm{mol}^{-1}$ was selected as the target line. ${ }^{26}$ The temperature of the DL3 was set to $27^{\circ} \mathrm{C}$ with an output power of $10.0 \mathrm{~mW}$, and the current was scanned from $100 \mathrm{~mA}$ to $140 \mathrm{~mA}$, corresponding to a tuning range between $6542.63 \mathrm{~cm}^{-1}$ and $6541.29 \mathrm{~cm}^{-1}$. A gas mixture of $500 \mathrm{ppm} \mathrm{C}_{2} \mathrm{H}_{2}$ and $1.6 \%$ water vapor in pure $\mathrm{N}_{2}$ at 700 Torr pressure was used in the gas cell. The wavelengths of the DL1 and DL3 were spectrally scanned in periods of $60 \mathrm{~s}$ and $100 \mathrm{~s}$ corresponding to scan rates of $0.024 \mathrm{~cm}^{-1} / \mathrm{s}$ and $0.0067 \mathrm{~cm}^{-1} / \mathrm{s}$, respectively. The LIAs settings were not altered. The detection bandwidth of the QEPAS sensor system was $0.833 \mathrm{~Hz}$. The measured spectral scans are shown in Fig. 5. The $2 f$ spectra of $\mathrm{H}_{2} \mathrm{O}$ and $\mathrm{C}_{2} \mathrm{H}_{2}$ were acquired every $60 \mathrm{~s}$ and $100 \mathrm{~s}$, respectively, without signal cross-talking. To compare the sensor performance of both detection channels, we calculated the normalized noise equivalent absorption (NNEA) coefficient for $\mathrm{H}_{2} \mathrm{O}$ and $\mathrm{C}_{2} \mathrm{H}_{2}$ to be $9.12 \times 10^{-7} \mathrm{~cm}^{-1} \cdot \mathrm{W} / \mathrm{Hz}^{1 / 2}$ and

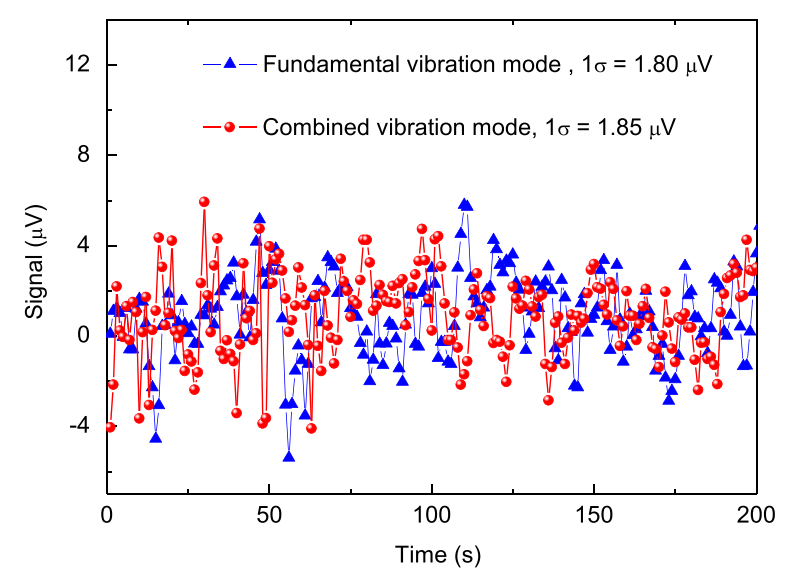

FIG. 4. Noise measurement of the QEPAS sensor operating in the pure fundamental flexural mode (blue triangle line) and in combined vibration motion (red rectangle and lines). 


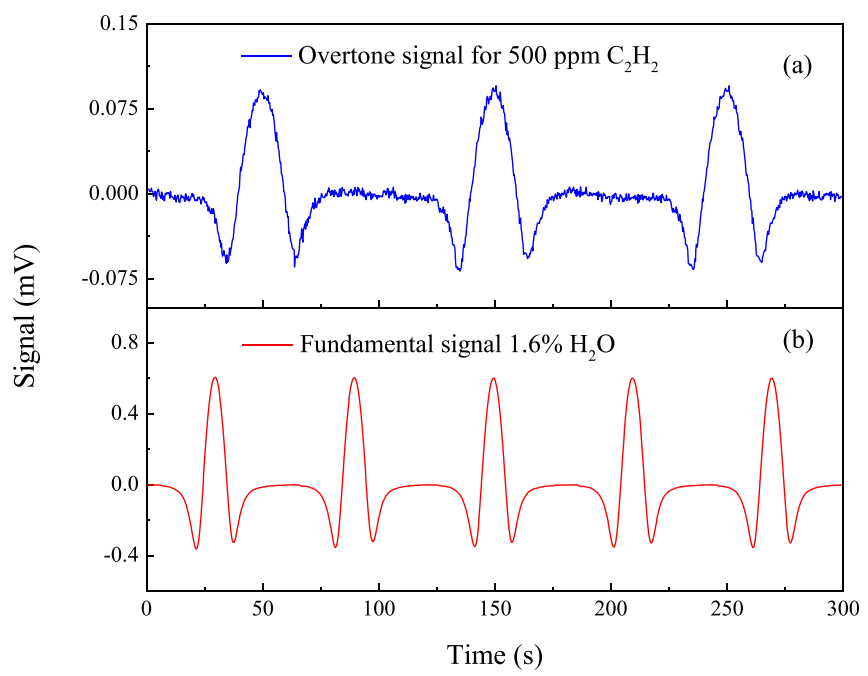

FIG. 5. Simultaneous dual-gas measurements using a QEPAS sensor based on combined vibration motion of a QTF. The optimized modulation depths for $\mathrm{C}_{2} \mathrm{H}_{2}$ and $\mathrm{H}_{2} \mathrm{O}$ detection were $0.51 \mathrm{~cm}^{-1}$ and $0.43 \mathrm{~cm}^{-1}$, respectively.

$1.51 \times 10^{-7} \mathrm{~cm}^{-1} \cdot \mathrm{W} / \mathrm{Hz}^{1 / 2}$, respectively. The detection sensitivity obtained from the detection channel of the 1st overtone frequency is $\sim 6$ times higher than that obtained for the detection channel of the fundamental frequency, which is in good agreement with previous reports on the single channel measurement. ${ }^{21,22}$

In conclusion, a dual-gas QEPAS sensor based on a QTF combined excitation of the fundamental and 1st overtone flexural modes was realized and analyzed. Essentially, the dual-gas signal measurements using the QTF combined vibration can be considered as a frequency division multiplexing (FDM) technique, allowing continuous real-time multi-gas monitoring with a compact sensor architecture. The two independent detection channels in such a QEPAS sensor permit optical signal addition or cancellation. This makes this method suitable for applications in controlling gas chemical reactions in order to optimize combustion processes. The proposed dual-gas sensing approach can be implemented when detecting gas with slow energy relaxation rates (such as $\mathrm{NO}, \mathrm{CO}$, and $\mathrm{CH}_{4}$ ). Typically, to enhance the QEPAS signals, relaxation promoters $\left(\mathrm{H}_{2} \mathrm{O}\right.$ or $\left.\mathrm{SF}_{6}\right)$ are added to the gas mixture, and thereby, it is also crucial to control their concentration. ${ }^{27}$ In a future development, the ratio between the detection sensitivities of the two independent detection channels can be adjusted by means of an appropriate design of the QTF prongs. ${ }^{22}$ Further improvements of the detection sensitivity can be achieved by adding dual- or single-tube acoustic micro-resonators (AmR) to enhance the acoustic wave intensity. ${ }^{19,28,29}$ A wider wavelength coverage can be realized when two widely tunable sources, e.g., optical parametric oscillator, are equipped as the exciting sources. 30,31

This material is based upon work supported by the National Natural Science Foundation of China (Grant Nos. 61622503 and 61575113). Frank Tittel acknowledges support by the National Science Foundation (NSF) ERC MIRTHE award and the Robert Welch Foundation (Grant No. C-0586). The authors from Dipartimento Interateneo di Fisica di Bari acknowledge financial support from two Italian research projects: PON02 00675 and PON02 00576.

${ }^{1}$ Y. Cao, N. P. Sanchez, W. Jiang, R. J. Griffin, F. Xie, L. C. Hughes, C. Zah, and F. K. Tittel, Opt. Express 23, 2121 (2015).

${ }^{2}$ Y. Yu, N. P. Sanchez, R. J. Griffin, and F. K. Tittel, Opt. Express 24, 10391 (2016).

${ }^{3}$ W. Ren, W. Jiang, and F. K. Tittel, Appl. Phys. B 117, 245 (2014).

${ }^{4}$ L. Dong, F. K. Tittel, C. Li, N. P. Sanchez, H. Wu, C. Zheng, Y. Yu, A. Sampaolo, and R. J. Griffin, Opt. Express 24, A528 (2016).

${ }^{5}$ M. Jahjah, W. Jiang, N. P. Sanchez, W. Ren, P. Patimisco, V. Spagnolo, S. C. Herndon, R. J. Griffin, and F. K. Tittel, Opt. Lett. 39, 957 (2014).

${ }^{6}$ H. Wu, L. Dong, X. Liu, H. Zheng, X. Yin, W. Ma, L. Zhang, W. Yin, and S. Jia, Sensors 15, 26743 (2015).

${ }^{7}$ J. Besson, S. Schilt, and L. Thévenaz, Spectrochim. Acta, Part A 63, 899 (2006).

${ }^{8}$ L. Dong, H. Wu, H. Zheng, Y. Liu, X. Liu, W. Jiang, L. Zhang, W. Ma, W. Ren, W. Yin, S. Jia, and F. K. Tittel, Opt. Lett. 39, 2479 (2014).

${ }^{9}$ M. Mordmüller, M. Köhring, W. Schade, and U. Willer, Appl. Phys. B 119, 111 (2015).

${ }^{10}$ N. Petra, J. Zweck, A. A. Kosterev, S. E. Minkoff, and D. Thomazy, Appl. Phys. B 94, 673 (2009).

${ }^{11}$ H. Yi, K. Liu, W. Chen, T. Tan, L. Wang, and X. Gao, Opt. Lett. 36, 481 (2011).

${ }^{12}$ Y. Cao, W. Jin, L. Ho, and Z. Liu, Opt. Lett. 37, 214 (2012).

${ }^{13}$ Y. Ma, X. Yu, G. Yu, X. Li, J. Zhang, D. Chen, R. Sun, and F. K. Tittel, Appl. Phys. Lett. 107, 021106 (2015).

${ }^{14}$ H. Wu, L. Dong, H. Zheng, X. Liu, X. Ying, W. Ma, L. Zhang, W. Yin, S. Jia, and F. K. Tittel, Sens. Actuators, B 221, 666 (2015).

${ }^{15}$ J. P. Waclawek, H. Moser, and B. Lendl, Opt. Express 24, 6559 (2016).

${ }^{16}$ Y. Ma, R. Lewicki, M. Razeghi, and F. K. Tittel, Opt. Express 21, 1008 (2013).

${ }^{17}$ L. Dong, J. Wright, B. Peters, B. A. Ferguson, F. K. Tittel, and S. McWhorter, Appl. Phys. B 107, 459 (2012).

${ }^{18}$ A. A. Kosterev, L. Dong, D. Thomazy, F. K. Tittel, and S. Overby, Appl. Phys. B 101, 649 (2010).

${ }^{19}$ H. Wu, A. Sampaolo, L. Dong, P. Patimisco, X. Liu, H. Zheng, X. Yin, W. Ma, L. Zhang, W. Yin, V. Spagnolo, S. Jia, and F. K. Tittel, Appl. Phys. Lett. 107, 111104 (2015).

${ }^{20}$ P. Patimisco, A. Sampaolo, L. Dong, M. Giglio, G. Scamarcio, F. K. Tittel, and V. Spagnolo, Sens. Actuators, B 227, 539 (2016).

${ }^{21}$ A. Sampaolo, P. Patimisco, L. Dong, A. Geras, G. Scamarcio, T. Starecki, F. K. Tittel, and V. Spagnolo, Appl. Phys. Lett. 107, 231102 (2015).

${ }^{22}$ F. K. Tittel, A. Sampaolo, P. Patimisco, L. Dong, A. Geras, T. Starecki, and V. Spagnolo, Opt. Express 24, A682 (2016).

${ }^{23}$ P. Patimisco, G. Scamarcio, F. K. Tittel, and V. Spagnolo, Sensors 14, 6165 (2014).

${ }^{24}$ K. Liu, X. Guo, H. Yi, W. Chen, W. Zhang, and X. Gao, Opt. Lett. 34, 1594 (2009).

${ }^{25} \mathrm{H}$. Yi, W. Chen, S. Sun, K. Liu, T. Tan, and X. Gao, Opt. Express 20, 9187 (2012).

${ }^{26}$ See http://www.hitran.com for HITRAN Database for the information about the spectral line parameter.

${ }^{27}$ X. Yin, L. Dong, H. Zheng, X. Liu, H. Wu, Y. Yang, W. Ma, L. Zhang, W. Yin, L. Xiao, and S. Jia, Sensors 16, 162 (2016).

${ }^{28}$ H. Zheng, L. Dong, A. Sampaolo, H. Wu, P. Patimisco, X. Yin, W. Ma, L. Zhang, W. Yin, V. Spagnolo, S. Jia, and F. K. Tittel, Opt. Lett. 41, 978 (2016).

${ }^{29}$ P. Patimisco, A. Sampaolo, H. Zheng, L. Dong, F. K. Tittel, and V. Spagnolo, Adv. Phys. X 2, 169 (2017).

${ }^{30}$ A. K. Y. Ngai, S. T. Persijn, I. D. Lindsay, A. A. Kosterev, P. Groß, C. J. Lee, S. M. Cristescu, F. K. Tittel, K. J. Boller, and F. J. M. Harren, Appl. Phys. B 89, 123 (2007).

${ }^{31}$ M. W. Sigrist, Rev. Sci. Instrum. 74, 486 (2003). 